Original Article

\title{
Insights from a Pilot Study of Naloxone Education
}

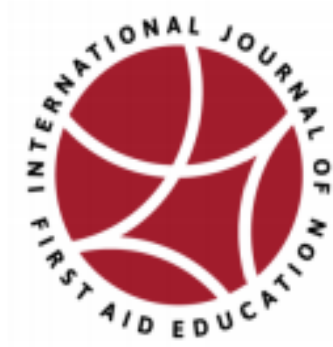

Tracey A. Taylor, Lucy Ellis, Penny Newell, Emily Oliver

Background and Aims: We wanted to test whether introducing an additional Naloxone component into overdose education would affect the willingness and confidence of a potential overdose witness to intervene effectively. A further aim was to test the potential of education to reduce barriers to administering Naloxone and barriers to calling 999 (UK Emergency services).

Methods: We used quantitative methods to gather and analyze data from both control (overdose education, $n=15$ ) and experimental (overdose and Naloxone education, $n=57$ ) participants, facilitated by educators in the North West of England and London, for opioid users $(n=5)$, former users $(n=19)$, carers/support workers $(n=29)$, family members $(n=31)$ or friends $(n=12)$ (multiple response item). We measured self-reported confidence and willingness of participants using evaluation forms with Likert scales, and knowledge using open-ended questions. We ran a focus group with educators about delivery, teaching and/or working with this study population as learners

Results: Both the controlled and the experimental sessions increased participants' confidence to use first aid skills, specific confidence in an overdose situation and willingness to act. The educators reported low levels of learner literacy, which may have affected the reliability of the data gathered. The sessions the benefits of a flexible, workshop-style session, peer-to-peer support.

Conclusion: Introducing a Naloxone component to first aid education targeting potential witnesses of an opioid overdose situation does not seem to negatively impact on learners' confidence or willingness to act, though the methodological challenges of this study limits the conclusiveness of this statement. Overdose or Naloxone education should employ a flexible format that is tailored to the needs of the learners and encourage peer-topeer support.

The number of heroin and/or morphine users in the United Kingdom (UK) is decreasing, from an estimated 94,000 users in $2011 / 12$ to 24,000 users in 2016/17 (UK Government Home Office Statistics, 2018). However, despite this, the number of deaths involving heroin and/or morphine increased, with the Office of National Statistics (ONS) in the UK documenting a rise from 579 deaths in 2012 to 1,164 deaths in 2017 (2018). Though the number of opioid-related deaths has risen each year to 2016, the rate of these deaths visibly plateaus between the years 2015 and 2016
(1201 deaths and 1209 deaths, respectively) and drops for the first time in 2017 (ONS, 2018). The ONS suggests that the plateau tracks the increased availability of naloxone. Naloxone is an opioid antagonist that reverses the effects of opioids in the brain and restores breathing (Gaston, Best, Manning, \& Day, 2009; Watt, Jaquet, Ellison, \& Nicholson, 2014). Following advice from the Advisory Council on the Misuse of Drugs (ACMD) the UK government made naloxone more widely available in 2015. 
In a survey covering $83.3 \%$ of local authorities in the UK ( $n=135)$, Public Health England and the Local Government Association (LGA) found that $90 \%$ of local authorities supplied take-home naloxone in 2017, a marked rise from 2015, when only a third of local authorities had elected to do so (Local Government Association, 2017). This same survey highlighted inconsistencies in the national roll-out of both take-home naloxone itself and naloxone education. The majority, 99\%, of local authorities provide naloxone through drug treatment services (Local Government Association, 2017). Although not a legal requirement, training is recommended (Public Health England, 2017). There is no standardized education offer, but Public Health England suggests that training covers topics including how to recognize an opioid overdose, overdose management and how to use the naloxone injection (Public Health England, 2017). This training is often brief, and the level of detail varies by provider.

Drug treatment service users and opioid users not in treatment represent the clear majority of people using local authorities to access naloxone $(95 \%$ of local authorities supply naloxone to drug treatment service users; $64 \%$ to opioid users not in treatment; and $79 \%$ to family/friends/carers of opioid users) (Local Government Association, 2017). ACMD and World Health Organization (WHO) guidelines (Advisory Council on the Misuse of Drugs, 2012; WHO, 2014) recommend that education on how to administer naloxone should be given to a broad non-medical audience to maximize the potential for this drug to save lives in cases of opioid overdose. The key justifications for this advocacy for naloxone education are clearly grounded in the literature. Most overdoses and overdose deaths are witnessed (Sporer, 2003; Strang, et al., 2008b; WHO, 2014). Overdose witnesses show a high rate of willingness to act (Strang, et al., 2008a; Seal et al, 2003). However, the actions of the witness are often ineffective, wrong, inefficient or inappropriate (Strang, Bird, \& Parmar, 2013). Learning how and when to administer naloxone can increase the confidence and knowledge of potential witnesses, making them more likely to act appropriately in an overdose situation (Gaston, Best, Manning, \& Day, 2009).

Many who might benefit from overdose and/or naloxone education are likely to be opioid users, potentially carrying or under the influence of an illegal psychoactive substance. Gaston, Best, Manning and Day (2009) found that opioid users were concerned about carrying naloxone on their person due to the stigma associated with carrying a needle (at the time of writing, in the UK administration of naloxone is only available through intramuscular needles). This suggests that overdose and/or naloxone education needs to address needle stigma. This is not something known to be covered by any current naloxone education.

Critics have raised concerns that providing opioid users with naloxone could encourage and condone opioid use, creating a 'safety net' (ACMD, 2012; Doleac \& Mukherjee, 2018) effect leading to complacency about contacting the emergency services (Sporer, 2003). Orkin and Buchman (2017) have deftly dismissed these criticisms, arguing that it is not the duty of overdose education and naloxone distribution (OEND) programs to address the inequity of emergency health services and the inaccessibility of these services to activate a chain of ongoing care in the case of an overdose. Rather, overdose and/or naloxone education "should integrate bystander and first aid interventions for opioid overdose with a continuum of appropriate ambulance and other emergency care services" (p.309). Persuading opioid users that they have equal access to emergency services is part of this duty.

\begin{abstract}
Aims
Administering naloxone can reverse an opioid overdose and save lives. Though actions of witnesses are often ineffective or wrong (Strang, Bird, \& Parmar, 2013) there is a high rate of willingness to act (Strang, et al., 2008; Seal et al, 2003). Our concern was to test whether introducing a complex component such as how to administer naloxone would impact negatively on the willingness and confidence of a potential overdose
\end{abstract}


witness to intervene effectively, reducing their propensity to act (Bakke, Steinvik, Angel, \& Wisborg, 2016; Oliver, Cooper, \& McKinney, 2013; Van de Velde, et al., 2012). A further aim was to test the potential of overdose or naloxone education to reduce barriers to administering naloxone, such as 'needle stigma' (Gaston, Best, Manning, \& Day, 2009) and barriers to calling 999 (UK Emergency services) (Orkin \& Buchman, 2017). We used quantitative methods to gather and analyze data from both a control (overdose) and experimental (overdose and naloxone) session, measuring self-reported confidence and willingness of participants using evaluation forms with Likert scales, and measuring knowledge using open-ended questions. We also ran a focus group to ascertain how educators utilized the resources, and if the educators found any barriers to delivery, teaching and/or learning. Our qualitative findings are presented alongside the educational outcomes as considerations for designing effective overdose education and/or naloxone education.

\section{Methods}

Control and experimental sessions were delivered by first aid educators working for the British Red Cross, in the Crisis Education department of the organization, with four control and seven experimental sessions taking place the North West of England and London. All 11 were face-to-face, group sessions, occurring between November 2016 and July 2017. Educators attended a one-day workshop prior to the sessions taking place at which they were introduced to the study and attended follow up meetings and conference calls to support their involvement during and after the study took place.

The study population was identified by local educators using their pre-established links with drug and alcohol support agencies. Participants were identified as being those most likely to encounter overdose situations. This included family members, support workers and opioid users, following in the footsteps of previous studies (Strang, et al., 2008b; Seal et al, 2003; Sporer, 2003; WHO, 2014). The agencies were responsible for recruitment of participants to the project, promotion and hosting the sessions. The sessions were provided free of charge. As the responsibility for recruitment and scheduling of sessions lay with the partner agencies, there was no formalized delivery schedule. The educators were responsible for delivering control and experimental sessions. Educators were allowed flexibility with regard to the type of session delivered (control or experimental, defined below), duration and style of delivery.

Personal information and responses provided by learners was held confidentially. Learners' demographic categorization was completed individually. The educators chosen were experienced at working with a range of audiences and utilized pedagogical methods to create a learning environment of mutual respect in line with the British Red Cross educational approach.

The research team designed the control session as a combination of first aid skills, including:

1. How to recognize an overdose

2. Helping someone who is unresponsive and not breathing

3. Helping something who is bleeding heavily

4. Helping someone who has taken something harmful

5. How to overcome the fear of calling 999 (UK Emergency Services)

6. Helper attributes

The research team initially developed the experimental resources working collaboratively with Martindale Pharma, the company which manufactures naloxone (sold as Narcan $($ ) ) as a prepackaged syringe, and which supplied the materials for the session. These resources, which were initially expected to be delivered in a two-hour session, were revised following feedback from educators regarding accessibility of information for use in the sessions. The experimental session comprised of both overdose and naloxone education, covering (1-6) the first aid skills outlined above and:

7. When and how naloxone is given (including a demonstration and 
practice using needles to inject oranges)

8. Information to address any potential resistance to carry naloxone (e.g. needle stigma)

Both sessions utilized British Red Cross educational methodology based on the principles that first aid should be easy learn, easy to do and easy to remember, and positioned as relevant to the learner. The learning objectives were to increase learners' confidence and willingness to act in an overdose situation, as well as removing the barriers to act including calling 999 and needle stigma.

The research team designed pre- and post-learning evaluation forms, to be distributed to participants before and after the control and experimental sessions. These forms included statements for participants to self-identify their demographic status, and three closed questions containing Likert scales of 0 (not at all) to 10 (extremely) as outlined below:

Q1. Thinking about the first aid skills you have just learned; how confident do you feel that you can use these skills effectively in an emergency? [general confidence]

Q2. How willing are you to use these skills in an emergency? [willingness]

Q3. Arriving at a friend's house, you find them unresponsive lying on the floor. Their breathing is very slow, their skin, fingers and face are pale, and you suspect they have been taking heroin. How confident do you feel that you could help the person in this situation? [confidence specific to overdose situation]

Data was anonymized and coded numerically, to be analyzed by two supporting researchers. Significance testing was run using SPSS (IBM SPSS Statistics, v24), however we also utilized an internal tool developed to take account of differences in pre-learning scores. This calculation used routinely by the British Red Cross to evaluate effectiveness of our education, is called Average Potential (AP) (Rothman, 2016). AP provides an indication of how effective each session was at helping each participant to reach their full potential for learning. For a single participant AP is obtained by calculating the difference in points between his or her pre-learning score for confidence/willingness and 10; then using his or her post-learning score to identify the change in confidence/willingness; and finally, expressing this change as a percentage of potential-for-change value.

Open-ended questions recorded knowledge concerning overdose recognition, and appropriate response to an overdose situation.

A focus group of educators involved in creating the materials and delivering the sessions was convened after the sessions had been held to collect feedback on the materials and to explore their reflections on any barriers to learning that they had experienced. This was an unplanned addition to the study, arising on the request of the educators involved. Their discussion was not analyzed as part of the study, although the themes they raised provided insight and context to the quantitative data. These themes are reported on in the results.

\section{Results}

\section{Demographics}

Table 1 shows the demographic split: participants self-identified their status and were able to select more than one statement of identity.

\section{Session information}

The seven experimental sessions had an average of nine participants (ranging from five to 14 participants). The sessions lasted an average of 125 minutes (ranging from 124-140 minutes).

The four control sessions had an average of four participants (ranging from two to eight participants). The sessions lasted an average of 70 minutes (ranging from 60-80 minutes).

No control sessions took place in the North West location. In London, four sessions were control and three were experimental.

\section{Learners' confidence and willingness}

Tests for normality and a visual inspection of the histograms revealed the data to be negatively skewed for all three questions in post-learning responses and therefore unsuited to analysis using parametric procedures. 
An analysis of 71 learners (14 control, 57 experimental) identified a difference between control and experimental groups for post-learning confidence to give first aid and post-learning confidence to give first aid in a specific overdose scenario. There was no difference however for post-learning willingness to give first aid, although both experimental and control groups increased from pre-learning levels. Table 2 shows the average confidence and willingness of the experimental and control session participants.

Table 1: Self-Demographic Statement

\begin{tabular}{|l|l|}
\hline \multicolumn{1}{|c|}{$\begin{array}{c}\text { Self-identified Demographic } \\
\text { Statement* }\end{array}$} & number \\
\hline $\begin{array}{l}\text { I use heroin and/or drugs like } \\
\text { codeine, morphine and } \\
\text { methadone }\end{array}$ & $\mathrm{n}=5$ \\
\hline $\begin{array}{l}\text { I have previously used heroin } \\
\text { and/or drugs like codeine, } \\
\text { morphine and methadone }\end{array}$ & $\mathrm{n}=19$ \\
\hline $\begin{array}{l}\text { I work with users of heroin/or } \\
\text { drugs like codeine, morphine and } \\
\text { methadone }\end{array}$ & $\mathrm{n}=29$ \\
\hline $\begin{array}{l}\text { I have a family member who uses } \\
\text { heroin/or drugs like codeine, } \\
\text { morphine and methadone }\end{array}$ & $\mathrm{n}=12$ \\
\hline $\begin{array}{l}\text { I have a friend who uses heroin /or } \\
\text { drugs like codeine, morphine and } \\
\text { methadone }\end{array}$ & $\mathrm{n}=31$ \\
\hline N/A (none selected) & $\mathrm{n}=12$ \\
\hline
\end{tabular}

Table 2: Average Pre/Post Scores (out of 10)

\begin{tabular}{|l|l|l|}
\hline Experimental group & Pre & Post \\
\hline Confidence average & 5.8 & 8.5 \\
\hline Willingness average & 7.2 & 8.8 \\
\hline Confidence specific average & 6 & 8.7 \\
\hline Control group & Pre & Post \\
\hline Confidence average & 5.4 & 8.5 \\
\hline Willingness average & 7.1 & 9 \\
\hline Confidence specific average & 5.2 & 8.5 \\
\hline
\end{tabular}

Experimental groups were found to have a higher mean ranking for post-learning confidence to give first aid (control=26.5; experimental=38.3). A Mann Whitney $\mathrm{U}$ test found the difference between the two categories to be statistically significant $(\mathrm{U}=266.5, \mathrm{df}=1, \mathrm{p}=0.045)$. Experimental groups were found to have a higher mean ranking for post- learning confidence to give first aid in a specific overdose scenario (control $=26.9$; experimental $=$ 38.3). A Mann Whitney $U$ test found the difference between the two categories to be slightly above the prescribed level of statistical significance $(\mathrm{U}=271$, $\mathrm{df}=1, \mathrm{p}=0.051)$ and therefore no significant difference between control and experimental courses can be claimed for confidence to give first aid in an overdose scenario.

\section{Average potential}

Comparing the Average Potential of the experimental and control sessions, the experimental session was more effective at increasing both confidence (67\% compared to $49 \%$ control) and willingness (67\% compared to 55\% control). For the question asking about confidence to help in a specific overdose scenario, AP was 51\% for the control compared to $64 \%$ for the experimental session.

\section{Correlation with session length}

It is important to remember that the experimental sessions had a longer duration. An increase in the length of the session was positively correlated $(\mathrm{r}=0.7)$ to increase in confidence (figure 1). Consequently, it is not possible to discern whether the increase in confidence was due to the length of session or to the content of the experimental session resources. Length of session did not correlate with an increase in willingness.

\section{Qualitative results}

Qualitative data were sought to provide context to inform and build on the quantitative findings and understand validity.

The open-ended knowledge question asked of participants needed to be abandoned due to lack of comprehension and ability to respond. Responses that were received could not be adequately coded to be of use in this instance.

The focus group of educators involved in creating the materials and delivering the sessions took place after the sessions had been held to collect feedback on the materials and to explore their reflections on any barriers to learning that they had experienced. In the course of the discussion, the following topics were raised: 


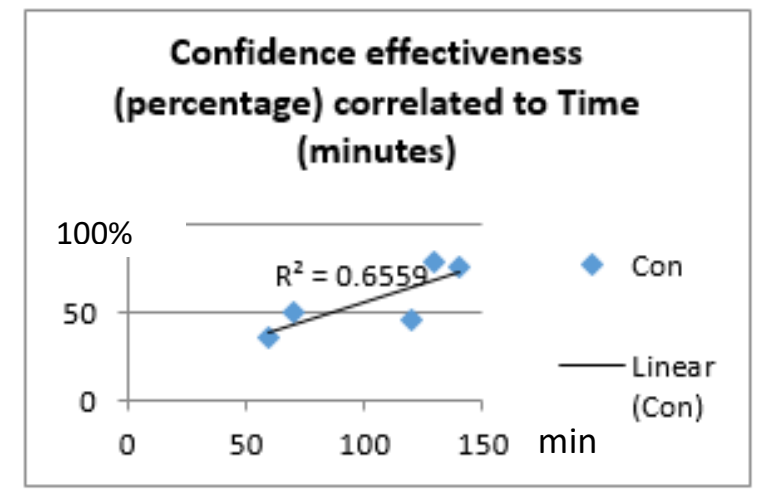

Figure 1: Confidence Difference

\section{Stigma and fear}

The experimental session included a stigma activity; however, the educators did not identify needle stigma as an area for concern with the participants. The educators confirmed that the participants had expressed fears about alerting the emergency services.

\section{Procedure and materials}

Educators reported that some participants struggled to read the evaluation forms and voiced concerns about the inaccessibility of this evaluation method for participants. With smaller groups this was less of an issue: the educator could provide individual support to those with lower levels of literacy skills. The results drawn from the evaluation forms should be treated with caution due to the potential variation in levels of comprehension.

\section{Recruitment}

Partner drug and alcohol agencies were responsible for participant recruitment to the project. Due to the nature of the target learner group it was difficult to guarantee that a session would take place as planned, and that registered participants would actually attend. Educators emphasized the importance of having a good relationship with key workers in partner organizations, as they play a pivotal role in convincing participants to attend the sessions. Educators felt that simply advertising the session through a poster was not sufficient in generating attendance or interest. The educators reported struggling to deliver sessions in unsuitable rooms. They felt that the naloxone component benefitted from a central demonstration table using naloxone training kits and ample space in the room, to ensure safety using needles. (Educators reported no incidents with needles.)

The educators perceived an overall lack of national consistency around both naloxone distribution and training as a difficulty. It was their perception that areas where organizations are already working and delivering naloxone sessions are the easiest to target, due to prior local awareness of the importance of naloxone.

\section{Content}

In terms of the first aid skills taught, rescue breaths were included as part of resuscitation education in both control and experimental sessions, on account of the likelihood of respiratory arrest in cases of opioid overdose. The educators relayed participants' general reluctance to carry out rescue breaths.

\section{Potential revisions to delivery, duration and intensity}

The original session guide gave timings for each activity, but educators were given the flexibility to adapt these as they felt necessary based on the needs of their group of participants. Educators highlighted the importance of being able to do this to keep the participants engaged. The educators agreed that attention span was a potential issue for this study population.

\section{Approaching this study population as learners}

All the educators elected to run their sessions interactively. Although no guidance on session style was provided, educators had been chosen for their experience and learner-led approach. Educators described how participants related traumatic experiences of witnessing overdoses, or even of overdosing themselves, and agreed that participants were very open to talking about these experiences. Neither experimental nor control sessions were intended to provide psychosocial support for these issues. Nonetheless, all educators agreed that naloxone and/or overdose education has the potential to fulfil this aim by engendering a safe space of peer-to-peer support. This was found to be especially important when talking about 
calling the emergency services: the peer to peer feedback was felt to be more trustworthy than educator guidance on calling the emergency services.

Educators reported two main difficulties with attendance. Firstly, although participants were encouraged to attend and stay for the duration of the session, it was not compulsory, and so some may have left part way through. Secondly, educators had difficulties keeping the attention of participants. This was especially pertinent with smaller group sizes, as the group size limited the available activities. For example, some of the activities involved learners taking part in group discussions. Larger groups would benefit from a range of participants' views and brought more energy to the sessions.

Scheduling the sessions presented separate difficulties. Where participants were current opioid users, educators reported absence from a morning session, or attendance under the influence at an afternoon session. With little control over this, educators felt it to be a substantial barrier to learning.

\section{Discussion}

Tailoring first aid education for opioid users and those close to them has drawn out some important lessons which reflect the different domains in the Chain of Survival Behaviours (IFRC, 2016). The naloxone element adds a new layer to the type of 'preparedness' a learner might acquire through a regular first aid course or overdose education. The education itself (both control and experimental) utilized the domains of 'recognize', 'first aid' and 'access help' to provide the most relevant education for this particular population.

Factors that affect the propensity to act, from which we used confidence and willingness of a learner to intervene in a first aid emergency, influenced our research question. We wanted to explore whether introducing a complex component such as how to administer naloxone would impact negatively on the willingness and confidence of a potential overdose witness to intervene effectively, reducing their propensity to act. A further aim was to test the potential of overdose education to reduce barriers to action, including needle stigma and reluctance to call 999. Introducing a naloxone component to first aid education appears to increase our target learners' confidence or willingness to act.

Our participants were individuals with personal, traumatic experiences of witnessing or experiencing an overdose situation, similar to those studied by Strang, et al. (2008a). It seems logical that educators should anticipate these experiences and build a supportive environment to encourage peer-to-peer support within the session, and further research could focus on this gap in the evidence base. Anecdotally, this approach could include creating an atmosphere of respect, confidentiality and anonymity. A consideration for the future could be to include creating group charter for behavior during the session. This research project demonstrated the value of facilitating naloxone education in group, interactive sessions. There were interesting lessons for us as program designers regarding the benefit of incorporating hands on practice with needles and oranges and the complete absence of any safety issues arising. The value of group discussion was particularly demonstrated when participants spoke about calling 999 (UK Emergency Services). The feeling of being stigmatized by the emergency services (Orkin \& Buchman, 2017) could not be addressed by the educator alone; rather, a group dynamic gave participants the opportunity to share experiences and dispel each other's fears. This builds on findings that suggest overdoses are often witnessed by family carers (Strang, et al., 2008b), compounding this with the traumatic repercussions of the experience. The fact that our experimental sessions were longer than the control sessions, allowing therefore not only for additional activities, but also an inevitable opportunity for additional discussion (due to the learner-led training style consciously adopted by the educators), is likely to have affected the results, beyond the impact of the additional activities alone. Further research could explore the potential value of overdose education and naloxone training as a peer-to-peer support environment. New research could endeavor to explore why, even with prior education about 
overdose situations and experience of them, participants benefited from attending a session.

In our findings, attendance and attention span can be a problem, affecting group size and duration. Educators need to be equipped to deal with attention span issues arising in the study population and remain free to adjust session length and break times according to learner needs. Partnering with agencies and developing close relationships with key workers could help to address these potential issues of attendance, and support logistics such as time of day, location of training and room specifications. Resources need to be flexible enough to account for the volatility of group size, and accessible and varied enough to account for low levels of attention.

Although our study did not set out to explore whether teaching clinical first aid skills in isolation would be sufficient to change behavior in overdose situations, our results suggest that overdose education and naloxone training potentially offer a more effective approach to addressing the needs of the target audience. Confronting the reasons behind any fear of calling for help cannot be the responsibility of the educators exclusively but needs to be supported by de-stigmatizing policy and making emergency healthcare accessible to all. Overdose and naloxone education needs to address other social barriers, such as the reluctance to give rescue breaths. Our educators expressed that a more consistent national naloxone offer would help to support agencies and organizations, in attracting people to sessions. This emphasis on consistency supports the need for clear public health messaging about the life-saving potential of this anti-opioid drug, as one link in a chain of care that needs to be activated in an overdose situation. The effectiveness of naloxone distribution depends on consistency of national availability, consistent messaging and consistent education covering both clinical first aid skills and social behavioral factors.

\section{Limitations and opportunities}

Methodological challenges of this study limit the conclusiveness of our findings. Though actions of witnesses are often ineffective or wrong, (Strang,
Bird, \& Parmar, 2013) and first aid education should look to address these mistaken actions, our study cannot speak to the acclaimed increase of knowledge through overdose and/or naloxone training due to the lack of clarity around the knowledge measure. Our decision to assess knowledge using open ended questions proved to be inappropriate since the results could not be adequately coded or analyzed due to the literacy levels of the participants. Future research needs to identify other ways to evaluate the effectiveness of education using more inclusive methods.

This study adds to the current evidence base, but there are other ways in which this topic needs to be explored to maximize the benefits of naloxone as a lifesaving tool. This study was limited by a lack of prior knowledge of the specific demands of working with this study population. Regarding the invalidated knowledge results, had the research team anticipated low levels of literacy reported by the educators, we would have designed visual/kinetic knowledge tests. Without this comparator we cannot know if the validity of knowledge results is due to an ill-fitting written evaluation method, or due to poorly designed knowledge questions.

The flexibility of the study delivery paradoxically contributed both to the success and to the limitations of the study. It allowed the educators to change the intensity and duration of the sessions in accordance with levels of attention; to work closely with participants in smaller groups, to aid with completion of the evaluation form; and to deliver using a workshop-style format, allowing for a peerto-peer supporting environment. Flexibility facilitated the accessibility of the sessions and resources and enabled a key learning point regarding calling 999. The interactive style was useful for the participants but made it difficult to compare across the study sessions, as uniformity of discussion and delivery cannot be assumed. Participants in smaller groups with more guidance on completing the form may have been at an advantage. Our ability to draw conclusions from the quantitative data is limited by our inability to conclude how flexibility affected participants' comprehension and completion of the form. 
Educators had the flexibility to choose whether to deliver a control or experimental session with their groups. With hindsight, this was problematic as it led to a disproportionate number of the courses being experimental and no control sessions occurring in the North West.

\section{Disclosure}

Simulated Narcan ${ }^{\circledR}$ packs containing a syringe were provided, free of charge, by Martindale Pharma for this study. No financial support was given and Martindale Pharma did not influence the study design or participate in any of the control or experimental sessions in any way. Nonetheless, a limitation of this study was that other forms of provision of naloxone (such as a nasal naloxone) were not tested. This was because other forms of naloxone were not available in the UK at the time.

\section{Conclusion}

This study worked with a targeted study population. Future research could explore the general public's attitudes towards naloxone and their confidence and willingness to act in an overdose situation. This could provide insight into the value of incorporating a naloxone component into a public first aid offer. Within the UK this may be limited at the current time due to the inconsistencies in the national roll-out of naloxone provision. Further exploration into a more consistent, multi-agency approach could also be beneficial.

\section{Acknowledgements}

We would like to acknowledge: Daniel Colebourn, previously Education Researcher at the British Red Cross; Martin Tilbury, National Crisis Education Manager at the British Red Cross; educators Eric Lee, Carrie Reid, Grant Hoyle and Mike McGinley, for delivering the project; educators Jenny Jones, Hayley Walker and Paul Barnes, for contributing to the development workshops; and all the participants who contributed to this study.

\section{References}

Advisory Council on the Misuse of Drugs (ACMD). (2012). Consideration of Naloxone . ACMD. Retrieved from https://assets.publishing.service.gov.uk/government/uploads/system/uploads/attachment_data/file /119120/consideration-of-naloxone.pdf

Bakke, H. K., Steinvik, T., Angell, J., \& Wisborg, T. (2016). A nationwide survey of first aid training and encounters in Norway. BMC emergency medicine, 17(1), 6.

Doleac, J. L., \& Mukherjee, A. (2018). The moral hazard of lifesaving innovations: naloxone access, opioid abuse, and crime. IZA Discussion Papers, No. 11489, Institute of Labor Economics (IZA), Bonn

Gaston, R. L., Best, D., Manning, V., \& Day, E. (2009). Can we prevent drug related deaths by training opioid users to recognise and manage overdoses?. Harm Reduction Journal, 6(1), 26.

International Federation of the Red Cross (2016). International first aid and resuscitation guidelines 2016. Geneva, Switzerland.

Local Government Association. (2017). Naloxone Survey 2017. Retrieved from https://www.local.gov.uk/sites/default/files/documents/LGA\%20Naloxone\%20survey\%202017.pdf

Oliver, E., Cooper, J., \& McKinney, D. (2014). Can first aid training encourage individuals' propensity to act in an emergency situation? A pilot study. Emerg Med J, 31(6), 518-520.

Office of National Statistics. (2018). Deaths related to drug poisoning by selected substances in England and Wales: 2017 registrations. Retrieved from 
https://www.ons.gov.uk/peoplepopulationandcommunity/birthsdeathsandmarriages/deaths/datase ts/deathsrelatedtodrugpoisoningbyselectedsubstances Accessed on 04/04/2019

Orkin, A.M., \& Buchman, D. Z. (2017). Commentary on McAuley et al.(2017): Naloxone programs must reduce marginalization and improve access to comprehensive emergency care. Addiction, 112(2), 309-310.

Public Health England. (2017). Take-home naloxone for opioid overdose in people who use drugs. Public Health England. Retrieved from https://assets.publishing.service.gov.uk/government/uploads/system/uploads/attachment data/file 669475/phetake-homenaloxoneforopioidoverdoseaug2017.pdf

Rothman, J. (2016) Notes on Red Cross Data - provided for British Red Cross internal use by Pro Bono Economics (not available publicly)

Seal, K. H., Downing, M., Kral, A. H., Singleton-Banks, S., Hammond, J. P., Lorvick, J., ... \& Edlin, B. R. (2003). Attitudes about prescribing take-home naloxone to injection drug users for the management of heroin overdose: a survey of street-recruited injectors in the San Francisco Bay Area. Journal of Urban Health, 80(2), 291-301.

Sporer, K. A. (2003). Strategies for preventing heroin overdose. BMJ: British Medical Journal, 326(7386), 442.

Strang, J., Bird, S. M., \& Parmar, M. K. (2013). Take-home emergency naloxone to prevent heroin overdose deaths after prison release: rationale and practicalities for the N-ALIVE randomized trial. Journal of Urban Health, 90(5), 983-996.

Strang, J., Manning, V., Mayet, S., Best, D., Titherington, E., Santana, L., ... \& Semmler, C. (2008a). Overdose training and take-home naloxone for opiate users: prospective cohort study of impact on knowledge and attitudes and subsequent management of overdoses. Addiction, 103(10), 1648-1657.

Strang, J., Manning, V., Mayet, S., Titherington, E., Offor, L., Semmler, C., \& Williams, A. (2008b). Family carers and the prevention of heroin overdose deaths: Unmet training need and overlooked intervention opportunity of resuscitation training and supply of naloxone. Drugs: education, prevention and policy, 15(2), 211-218.

UK Government Home Office Statistical Bulletin 1418 (2018) Drug Misuse: findings from the 2017-2018 SEW Ref: ISBN 978-1-78655-690-5 Retrieved from https://www.gov.uk/government/statistics/drugmisuse-findings-from-the-2017-to-2018-csew Accessed on 04/04/2019

Van de Velde, S., Roex, A., Vangronsveld, K., Niezink, L., Van Praet, K., Heselmans, A., ... \& Aertgeerts, B. (2013). Can training improve laypersons helping behaviour in first aid? A randomised controlled deception trial. Emerg Med J, 30(4), 292-297.

Watt, G., Jaquet, S., Ellison, S., \& Nicholson, J. (2014). Service Evaluation of Scotland's Take-home Naloxone programme. Retrieved from https://www.gov.scot/publications/service-evaluation-scotlands-takehome-naloxone-programme/pages/11/

World Health Organization, Management of Substance Abuse Unit. (2014). Substance use: Community management of opioid overdose. Retrieved from https://www.who.int/substance abuse/publications/management opioid overdose/en/ 